\title{
Genotype-dependent differences in age of manifestation and arrhythmia complications in short QT syndrome ${ }^{\text {th }}$
} \author{
Makoto Takano $^{\mathrm{j}}$, Silvia G. Priori ${ }^{\mathrm{c}, \mathrm{k}}$, Naomasa Makita ${ }^{\mathrm{a}, *}$ \\ a Department of Molecular Physiology, Nagasaki University Graduate School of Biomedical Sciences, Nagasaki, Japan \\ b Department of Cardiovascular and Respiratory Medicine, Shiga University of Medical Science, Heart Rhythm Center, Shiga, Japan \\ c Molecular Cardiology, IRCCS Salvatore Maugeri Foundation, Pavia, Italy \\ ' Department of Pediatric Cardiology, Okinawa Children's Medical Center, Okinawa, Japan \\ e Second Department of Internal Medicine, University of Occupational and Environmental Health, Kitakyushu, Japan \\ ${ }^{\mathrm{f}}$ Department of Heart Rhythm Management, University of Occupational and Environment Health, Kitakyushu, Japan \\ ${ }^{g}$ Department of Cardiovascular Medicine, Nagasaki University Graduate School of Biomedical Sciences, Nagasaki, Japan \\ h Department of Pediatric Cardiology, Saitama International Medical Center, Hidaka, Japan \\ ${ }^{i}$ Sapporo Heart Center, Sapporo, Japan \\ j Department of Physiology, Kurume University School of Medicine, Kurume, Japan \\ k Department of Molecular Medicine, University of Pavia, Pavia, Italy
}

Daniel Toshio Harrell a , Takashi Ashihara b, Taisuke Ishikawa a , Ichiko Tominaga a,1, Andrea Mazzanti c Kazuhiro Takahashi ${ }^{\mathrm{d}}$, Yasushi Oginosawa ${ }^{\mathrm{e}}$, Haruhiko Abe ${ }^{\mathrm{f}}$, Koji Maemura ${ }^{\mathrm{g}}$, Naokata Sumitomo ${ }^{\mathrm{h}}$, Kikuya Uno ${ }^{\mathrm{i}}$,

\section{A R T I C L E I N F O}

\section{Article history:}

Received 7 February 2015

Received in revised form 3 April 2015

Accepted 14 April 2015

Available online 15 April 2015

\section{Keywords:}

Short QT syndrome

Mutation

Patch clamp

Meta-analysis

Computer simulation

\begin{abstract}
A B S T R A C T
Background: Short QT syndrome (SQTS) is a rare inheritable arrhythmia, associated with atrial and ventricular fibrillations, caused by mutations in six cardiac ion channel genes with high penetrance. However, genotypespecific clinical differences between SQTS patients remain to be elucidated.

Methods and results: We screened five unrelated Japanese SQTS families, and identified three mutations in KCNH2 and KCNQ1. A novel mutation KCNH2-I560T, when expressed in COS-7 cells, showed a 2.5-fold increase in peak current density, and a positive shift $(+14 \mathrm{mV}$ ) of the inactivation curve compared with wild type. Computer simulations recapitulated the action potential shortening and created an arrhythmogenic substrate for ventricular fibrillation. In another family carrying the mutation KCNQ1-V141M, affected members showed earlier onset of manifestation and frequent complications of bradyarrhythmia. To determine genotype-specific phenotypes in SQT1 (KCNH2), SQT2 (KCNQ1), and other subtypes SQT3-6, we analyzed clinical variables in 65 mutationpositive patients among all the 132 SQTS cases previously reported. The age of manifestation was significantly later in SQT1 (SQT1: $35 \pm 19$ years, $\mathrm{n}=30$; SQT2: $17 \pm 25$ years, $\mathrm{n}=8$, SQT3-6: $19 \pm 15$ years, $\mathrm{n}=15$; $\mathrm{p}=0.011$ ). SQT2 exhibited a higher prevalence of bradyarrhythmia (SQT2: 6/8, 75\%; non-SQT2: 5/57, 9\%; $\mathrm{p}<0.001$ ) and atrial fibrillation (SQT2: 5/8, 63\%; non-SQT2: 12/57, 21\%; $\mathrm{p}=0.012$ ). Of 51 mutation-positive individuals from 16 SQTS families, nine did not manifest short QT, but exhibited other ECG abnormalities such as atrial fibrillation. The resulting penetrance, $82 \%$, was lower than previously recognized.

Conclusion: We propose that SQTS patients may exhibit different clinical manifestations depending upon their genotype.
\end{abstract}

(c) 2015 Elsevier Ireland Ltd. All rights reserved.

\section{Introduction}

Short QT syndrome (SQTS $)^{2}$ is a rare, inheritable cardiac electrical disease characterized by a shortened corrected QT interval

\footnotetext{
is This author takes responsibility for all aspects of the reliability and freedom from bias of the data presented and their discussed interpretation.

* Corresponding author at: Department of Molecular Physiology, Nagasaki University Graduate School of Biomedical Sciences, 1-12-4 Sakamoto, Nagasaki 852-8523, Japan.

E-mail address: makitan@nagasaki-u.ac.jp (N. Makita).

1 Current address: NTT Medical Center Tokyo, Tokyo, Japan.

2 SQTS: short QT syndrome.
}

$(\mathrm{QTC})^{3}$ and associated with a risk of sudden cardiac death (SCD), ${ }^{4}$ ventricular fibrillation (VF), and atrial fibrillation (AF), but without structural abnormalities [1,2]. SQTS is characterized by accelerated cellular repolarization, owing to either an enhanced outward repolarizing potassium current or a reduced inward depolarizing calcium current. To date, mutations responsible for SQTS have been identified in six ion channel

\footnotetext{
${ }^{3}$ QTc: corrected QT interval.

${ }^{4}$ SCD: sudden cardiac death
} 
genes: KCNH2 (SQT1) [3], KCNQ1 (SQT2) [4], KCNJ2 (SQT3) [5], CACNA1C (SQT4) [6], CACNB2 (SQT5) [6], and CACNA2D1 (SQT6) [7]. Previous reports suggest that the penetrance of these mutations is extremely high in families with SQT1, SQT2, and SQT3 $[8,9]$.

Congenital long QT syndrome (LQTS) ${ }^{5}$ is another repolarization disorder, for which mutations in 13 genes have been reported. Extensive genetic studies over two decades have improved the risk stratification and management of patients with LQTS $[10,11]$. In contrast, much less information is available about the genotype-phenotype correlations and natural history of SQTS. A cohort study of the European SQTS registry has shown that KCNH2 mutation carriers (SQT1) show significantly shorter QT intervals and better response to hydroquinidine therapy as compared with non-SQT1 patients [12]. Conversely, a unique clinical phenotype characterized by neonatal AF and bradycardia was reported in SQT2 with the KCNQ1 mutation V141M [13]. These studies suggest that SQTS may have some genotype-specific characteristics; however, because of the small number of cases studied to date, a robust genotype-phenotype correlation has not been identified.

By genetic screening of five unrelated Japanese SQTS probands, we found three mutations including a novel KCNH2 mutation I560T, and previously reported mutations KCNH2-T618I [14] and KCNQ1-V141M [15]. SQT2 patients carrying the KCNQ1-V141M mutation exhibited earlier onset and frequent complications of bradyarrhythmia, a clinical scenario similar to that previously reported [13]. To explore the potential genotype-specific phenotypes underlying SQTS, we included 32 previously reported genotyped SQTS families [6-9,12-19], and further analyzed their clinical variables. We found that SQT1 patients exhibit later onset of manifestation and SQT2 patients have a higher prevalence of bradyarrhythmias and AF, implying that clinical characteristics of SQTS can differ by genotype as seen in LQTS.

\section{Methods}

\subsection{Study cohorts}

We studied five unrelated Japanese families consisting of 10 affected family members. The QT interval was corrected for heart rate using Bazett's equation (QTc $=\mathrm{QT} /(\sqrt{ }(\mathrm{RR}))$. The diagnosis of SQTS was made based on QTc $\leq 330 \mathrm{~ms}$ or QTc $<360 \mathrm{~ms}$ with presence of VF episode, pathogenic mutations, or family history of SQTS or SCD before age of 40 years [20]. All individuals who participated in the study gave written informed consent prior to genetic and clinical investigations in accordance with the standards of the Declaration of Helsinki and the local ethics committees at each participating institution. To further characterize the genotype-specific characteristics, we created an additional cohort, which consisted of 132 SQTS patients including five families (10 cases) from the current study, 33 families (61 cases) from a review by Gollob et al. [8], and an additional 36 SQTS families (61 cases) that have been previously reported and available on PubMed as of May 2014 [6-9,12-19] (Supplemental Table S1). A full description of the methods is available as supplementary information.

\subsection{Genetic analysis}

Genomic DNA was extracted from the blood by a standard protocol. All the exons of $K C N H 2, K C N Q 1$, and $K C N J 2$ were PCR amplified as previously described [21-23] (Supplemental Table S2). Direct sequencing was performed using the ABI Genetic Analyzer 3130 (Life Technologies, Carlsbad, CA).

\footnotetext{
${ }^{5}$ LQTS: long QT syndrome.
}

\subsection{Biophysical analysis of KCNH2-I560T}

Site-directed mutagenesis was performed using the QuikChange site-directed mutagenesis kit (Agilent Technologies, Santa Clara CA) on human $\mathrm{KCNH} 2$ cDNA cloned in an expression plasmid pcDNA3.1 (Life Technologies). Oligonucleotide sequences are available in Supplemental Table S2. The COS-7 cell line was transiently transfected with wild-type (WT) or I560T-KCNH2 plasmid, and the potassium current was recorded by whole-cell patch-clamp techniques as previously described [24] with modifications.

\subsection{Western blotting}

To test the cell surface expression and the glycosylation of the I560T mutant channel protein, we carried out Western blotting analysis using WT channel and a neighboring LQTS mutation A561V as a control which is characterized by the membrane trafficking defect [25]. HeLa cells were transfected with $\mathrm{KCNH} 2$ plasmid of either WT, I560T, or A561V and the proteins extracted from the cell lysate were subjected to Western blotting as described previously [26]. Anti-KCNH2 polyclonal antibodies (1:400, Life Technologies) and anti- $\beta$ actin polyclonal antibodies (1:200, Cell Signaling Technology, Danvers, MA) were used as primary antibodies, and the signals were visualized by ECL Prime Detection Reagent (GE Healthcare).

\subsection{Computer simulation of action potential shortening and inducibility of lethal ventricular arrhythmia in KCNH2-I560T}

Computer simulations were carried out to determine whether the gating abnormalities and the increased current density of the $\mathrm{KCNH} 2$ I560T are sufficient to cause shortening of the action potential duration $(\mathrm{APD})^{6}$ and QT interval. Membrane kinetics were represented by the O'Hara-Rudy dynamic human ventricular model with modified Markovian $\mathrm{I}_{\mathrm{Kr}}$ equations [27-29], and the pseudo-ECG was calculated as described previously [30]. To demonstrate the relative arrhythmogenicity of KCNH2-I560T, we conducted simulations of VF in the bidomain endocardial sheet, and an S1-S2 cross-field protocol was applied to induce a spiral wave reentry.

\subsection{Clinical characteristics of SQTS with different genotypes}

In the study cohort of 132 SQTS patients, 65 patients were mutationpositive ( 6 from the current study and 59 from previous reports [6-9, $12-19])$ of whom 30 (46\%) were male and had a mean age of manifestation of $28 \pm 20$ years (ranging from 0 to 72 ). Clinical variables for each reported patient were extracted for sex, age of manifestation, QT, QTc, heart rate (HR), causative gene and mutation, and clinical history: AF, $\mathrm{SCD} /$ cardiac arrest, palpitations/syncope, and sick sinus syndrome/ bradycardia. Bradycardia for adult patients was evaluated based on $\mathrm{HR}<50 \mathrm{bpm}$. For children, bradycardia was determined based on $\mathrm{HR}<100 \mathrm{bpm}$ for ages 0 to 3 years and on $\mathrm{HR}<60 \mathrm{bpm}$ for ages 3-9 years [31]. A patient was considered symptomatic in the presence of the aforementioned clinical episodes.

\subsection{Statistical analysis}

Data are reported as mean \pm SD and analyzed by one-way ANOVA with Bonferroni correction. The univariate clinical variables are presented as percentages, and analyzed by $\chi^{2}$-test or Fisher exact test. Statistical significance was set at $\mathrm{p}<0.05$.

\footnotetext{
${ }^{6}$ APD: action potential duration.
} 
A Family 1
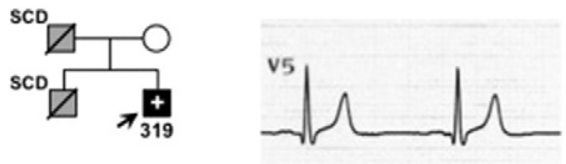

C Family 3

PPM
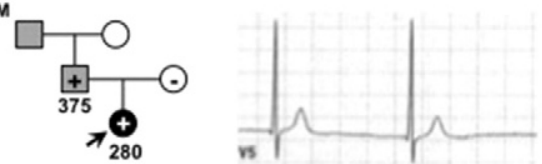

B Family 2

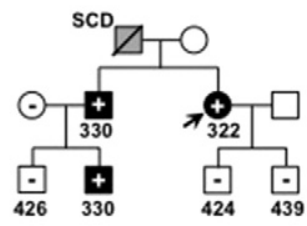

D Family 4

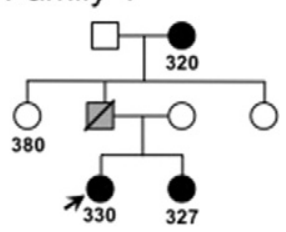

v5

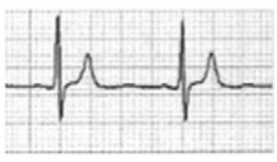

E Family 5
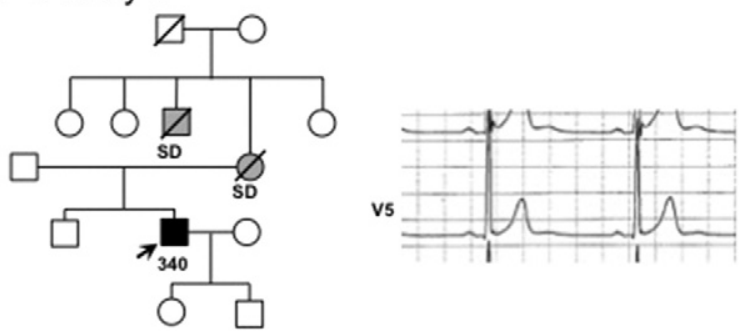

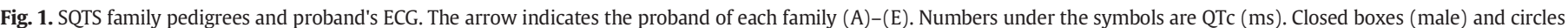

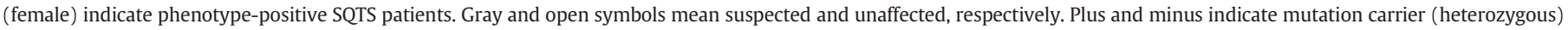
and non-carrier, respectively. SCD: sudden cardiac death, SD: sudden death, PPM: permanent pacemaker. V5 lead ECG of each proband is shown.

\section{Results}

3.1. Case presentation and genetic analysis of SQTS families (Figs. 1 and 2 and Table 1)

\subsubsection{Family 1}

A 64 year-old man experienced palpitations and near syncope due to paroxysmal AF and atrial flutter, for which he underwent catheter ablation (Fig. 1A). After the catheter ablation, short QT (QTc $=319 \mathrm{~ms}$, $\mathrm{HR}=68 \mathrm{bpm}$ ) with peaked $\mathrm{T}$ waves on the precordial leads became manifested, and he was diagnosed with SQTS (Supplemental Fig. S1). His father and brother died suddenly from unknown causes. Genetic screening revealed a novel heterozygous missense mutation in exon 7 of $K C N H 2$ (c.1679T > C), resulting in the amino acid substitution I560T, located in transmembrane segment S5 (Fig. 2A). KCNH2-I560T was absent in the genomic DNA of 200 healthy Japanese individuals, and in the public databases: dbSNP; 1000 Genomes project; Exome Variant Server; and Human Genetic Variation Database. The amino acid residue I560 is $100 \%$ conserved among eight different species (Fig. 2B). The proband has no offspring, and declined insertion of an implantable cardioverter defibrillator (ICD).

\subsubsection{Family 2}

A 39-year-old woman with aborted VF was diagnosed with SQTS (QTc $=322 \mathrm{~ms}, \mathrm{HR}=74 \mathrm{bpm}$ ) and had an ICD implanted (Fig. 1B, Supplemental Fig. S1, B). Combined prescription of bepridil with bisoprolol successfully prolonged her QTc to $341 \mathrm{~ms}$, and she has been free from VF attack for 2 years. Her father died suddenly from unknown causes at the age of 40 years and her brother and nephew exhibited short QTc (330 ms). Genetic screening identified a heterozygous missense mutation T618I (c.1853C > T) located in the pore region. This mutation was detected in all affected members (Fig. 2C). This mutation was previously reported [14].

\subsubsection{Family 3}

The index patient, a 10-year-old girl, was diagnosed with congenital sick sinus syndrome $(\mathrm{SSS})^{7}$ due to fetal bradycardia $(\mathrm{HR}=72 \mathrm{bpm})$ at the gestational age of 22 weeks (Fig. 1C). She was born by cesarean section at the age of 37 weeks, and exhibited sinus bradycardia $(\mathrm{HR}=$ $50 \mathrm{bpm}$ ) with bradycardia-induced heart failure, although echocardiography showed no organic heart diseases. As a result of a complete atrioventricular block at the age of 12 days, a permanent pacemaker was implanted. At 4 years of age, the patient demonstrated severe QT shortening $(\mathrm{QTc}=280 \mathrm{~ms})$ with bradycardia $(\mathrm{HR}=64 \mathrm{bpm})$ (Supplemental Fig. S1, C). Genetic screening revealed a heterozygous missense mutation V141M (c.421G >A) of KCNQ1 located in transmembrane segment S1 (Fig. 2D). This mutation was previously reported [15]. Her father had experienced chronic AF with bradycardia since the age of 3 years, but recorded a QTc value of $375 \mathrm{~ms}(\mathrm{HR}=37 \mathrm{bpm})$ by ECG, which is outside the diagnostic criteria of SQTS (Supplemental Fig. S1, D) [20]. Interestingly, despite this, he carried the V141M mutation. Her paternal grandfather had a pacemaker implanted at the age of 50 years, but declined genetic testing.

\subsubsection{Family 4}

A 17-year-old woman who survived an episode of VF was diagnosed with SQTS (QTc $=330 \mathrm{~ms}, \mathrm{HR}=83 \mathrm{bpm}$ ), and an ICD was implanted (Fig. 1D, Supplemental Fig. S1, E). Her sister and grandmother also displayed short QT. Genetic screening was negative.

\subsubsection{Family 5}

A 42-year-old man exhibited short QT (QTc $=340 \mathrm{~ms}$, HR = $53 \mathrm{bpm}$ ) without presence of organic heart disease (Fig. 1E). Covedtype ST elevation on V1 and V2 was observed after intravenous

\footnotetext{
7 SSS: sick sinus syndrome.
} 
A

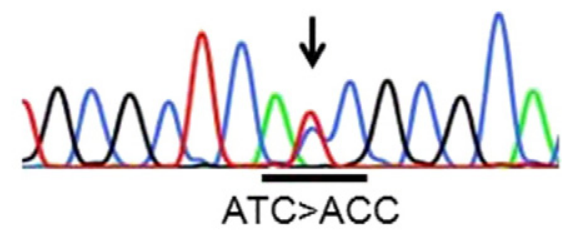

$\mathrm{KCNH} 2$
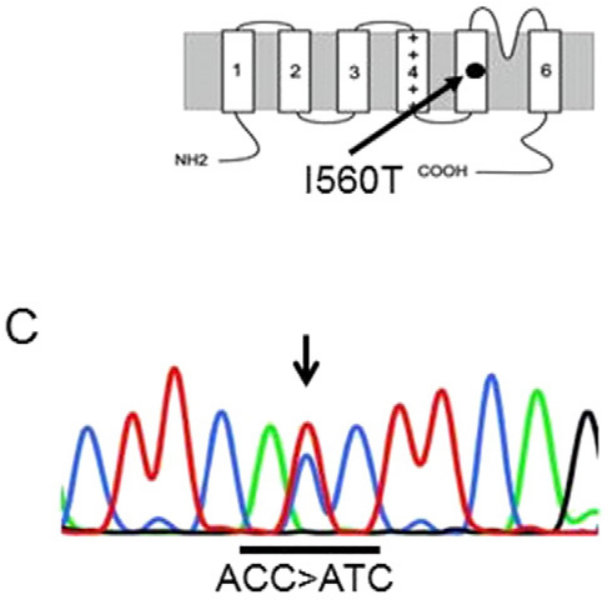

KCNH2

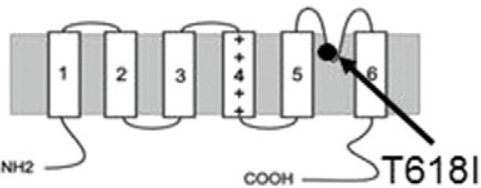

B

\begin{tabular}{lc} 
& \multicolumn{1}{c}{560} \\
Human & MCTFALIAHWLACIW \\
Chimp & MCTFALIAHWLACIW \\
Macaca & MCTFALIAHWLACIW \\
Rat & MCTFALIAHWLACIW \\
Canine & MCTFALIAHWLACIW \\
Bovine & MCTFALIAHWLACIW \\
Chicken & MCTFALIAHWLACIW \\
Zebrafish & \\
I560T & MCTFALT AHWLACIW
\end{tabular}

$\mathrm{D}$

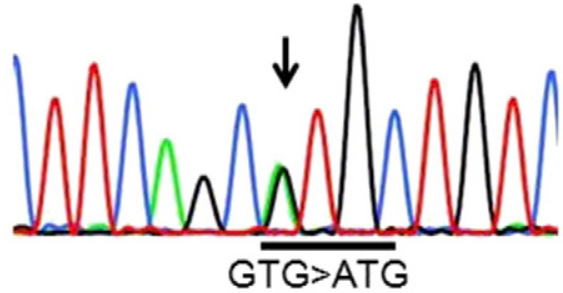

$K C N Q 1$

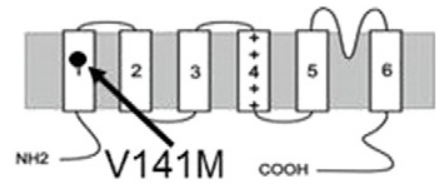

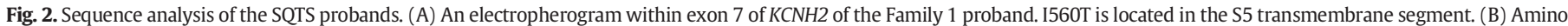

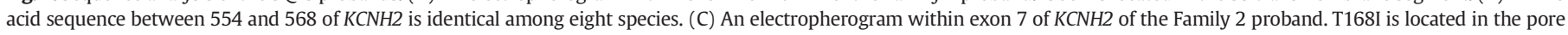
region of $K C N H 2$. (C) An electropherogram within exon 3 of KCNQ1 of the Family 3 proband. V141M is located in the S1 transmembrane segment.

administration of pilsicainide, indicating a complication of Brugada syndrome (Supplemental Fig. S1, F). A programmed electrical stimulation failed to induce VF. His mother died at 43 years and an uncle died suddenly from unknown causes. Genetic testing was negative for all six SQTS genes, as well as Brugada syndrome candidate genes including SCN5A, HCN4, KCND3, KCNE3, SCN1B, SCN3B, SCN10A, and TRPM4. An ICD was implanted as a primary preventative measure.

\subsection{Electrophysiological properties of KCNH2-I560T}

KCNH2-I560T heterologously expressed in COS-7 cells resulted in a significant 2.5 -fold increase in the peak $\mathrm{I}_{\mathrm{Kr}}$ current density versus WT (I560T: $99.7 \pm 10.2 \mathrm{pA} / \mathrm{pF}$; WT: $40.6 \pm 10.4 \mathrm{pA} / \mathrm{pF} ; \mathrm{p}<0.005$ ) (Fig. 3A,B), whereas the voltage dependence of activation was comparable (I560T: $-19.7 \pm 3.2 \mathrm{mV}$; WT: $-18.5 \pm 1.6 \mathrm{mV}$; NS) (Fig. 3C).

Table 1

Summary of clinical characteristics of SQTS families.

\begin{tabular}{|c|c|c|c|c|c|c|c|c|}
\hline Family & Proband/family & Gender & Age of manifestation (year) & QTc (ms) & Mutations & Arrhythmias & Symptoms & Family history \\
\hline 1 & Proband & M & 64 & 319 & KCNH2-I560T & Paroxysmal AF, AFL & Palpitation, syncope & SCD \\
\hline \multirow[t]{3}{*}{2} & Proband & $\mathrm{F}$ & 39 & 322 & KCNH2-T618I & Aborted VF & Palpitation & SCD \\
\hline & Brother & M & 42 & 330 & & - & - & \\
\hline & Nephew & M & 14 & 330 & & - & - & \\
\hline \multirow[t]{2}{*}{3} & Proband & $\mathrm{F}$ & $4^{*}$ & 280 & KCNQ1-V141M & SSS, fetal bradycardia & - & PPM \\
\hline & Father & M & 37 & 375 & & Chronic AF, bradycardia & - & \\
\hline \multirow[t]{3}{*}{4} & Proband & $\mathrm{F}$ & 17 & 330 & Negative & Aborted VF & - & - \\
\hline & Sister & $\mathrm{F}$ & 19 & 327 & & - & - & \\
\hline & Grandmother & $\mathrm{F}$ & 77 & 321 & & - & - & \\
\hline 5 & Proband & M & 42 & 340 & Negative & $\mathrm{BrS}$ & - & SD \\
\hline
\end{tabular}

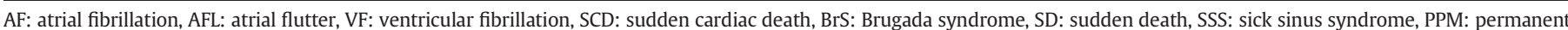
pacemaker.

*: Diagnosed at 10 years. Severe short QT (QTc $=280 \mathrm{~ms}$ ) demonstrated at 4 years. 
A
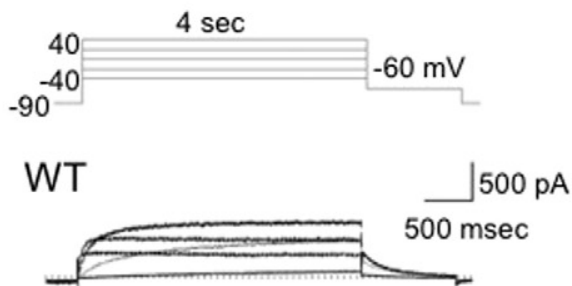

I560T

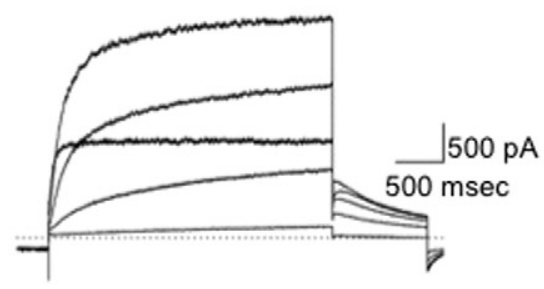

D

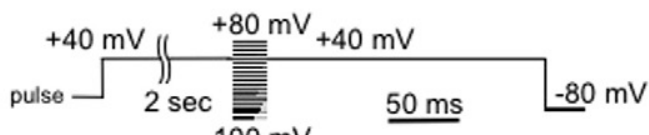

$-100 \mathrm{mV}$

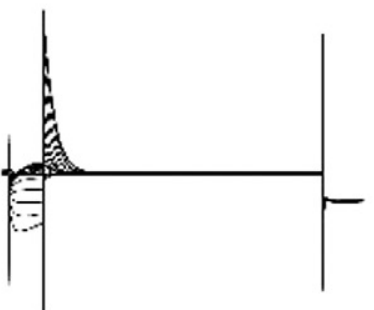

B

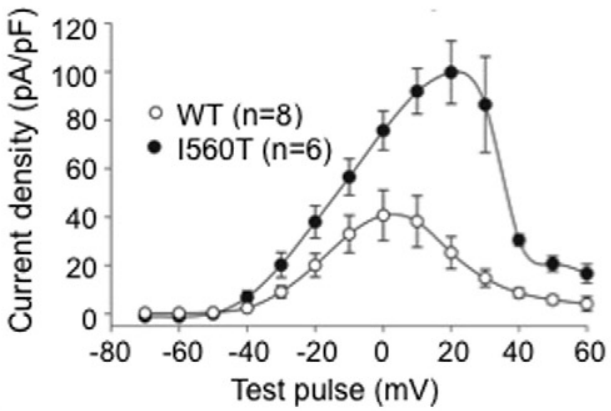

C

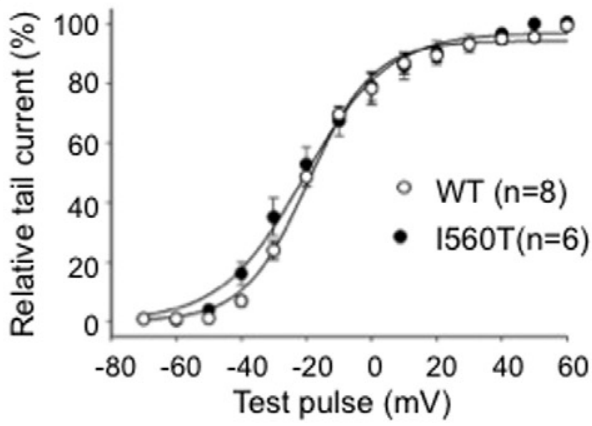

E

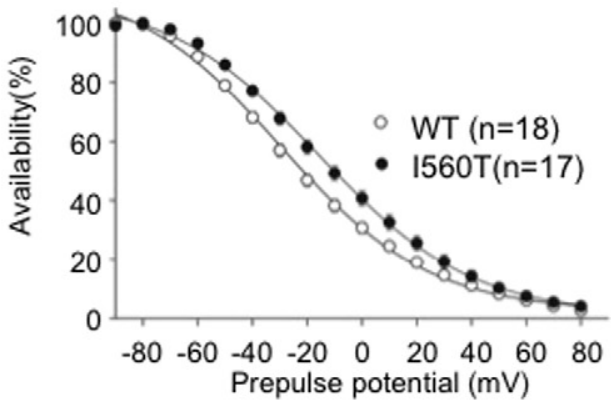

F

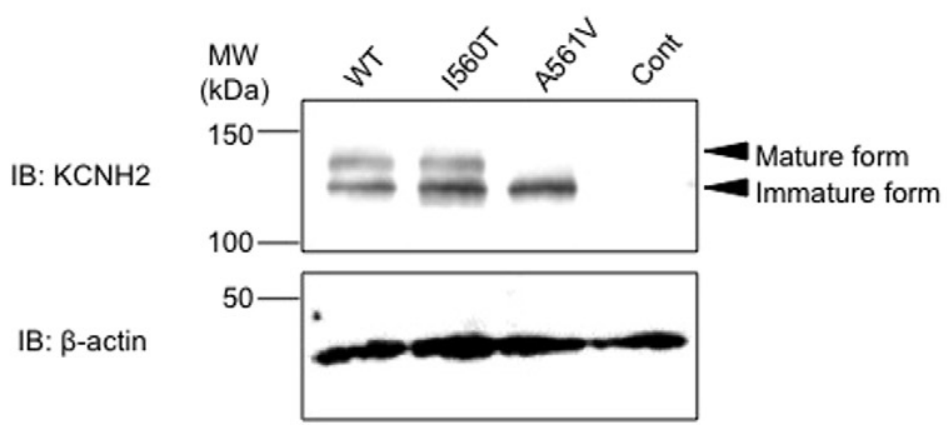

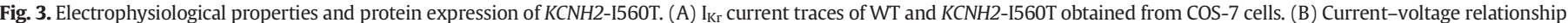

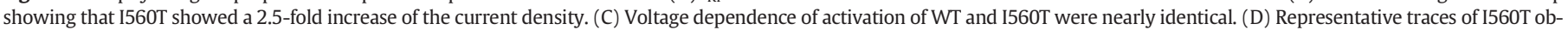

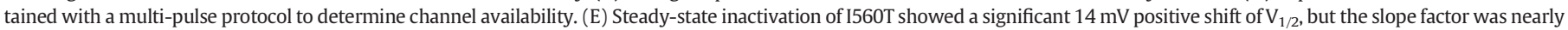
identical. (F) I560T expressed glycosylated mature protein at similar levels to WT. The trafficking-defective LQTS mutation A561V expresses unglycosylated immature proteins.

Steady-state inactivation showed a $14 \mathrm{mV}$ positive shift in the mutant channel (I560T: $-13.2 \pm 4.1 \mathrm{mV}$; WT: $-27.3 \pm 2.4 \mathrm{mV} ; \mathrm{p}<0.005$ ). The slope factor was nearly identical (I560T: $-26.5 \pm 1.2 \mathrm{mV}$; WT: $-25.4 \pm 1.1 \mathrm{mV}$; NS) (Fig. 3E). These results suggest that the mutant channel may cause a gain of function in $\mathrm{I}_{\mathrm{Kr}}$ current, which is a known trait of SQTS caused by KCNH2 mutations.

\subsection{Protein expression of $\mathrm{KCNH} 2-\mathrm{I} 560 \mathrm{~T}$}

To test if the $\mathrm{I}_{\mathrm{Kr}}$ gain of function observed in the KCNH2-I560T channel may be attributed to increased membrane expression levels or hyperglycosylation of the mutant channel protein, we performed Western blotting using a trafficking-defective neighboring $\mathrm{KCNH} 2$ mutation 
A561V as a control [25]. While the A561V mutant showed only minimal expression of mature glycosylated high-molecular-weight protein, the I560T mutant showed nearly identical expression levels and pattern to the WT protein (Fig. 3F). This confirms that the gain of function in $\mathrm{I}_{\mathrm{Kr}}$ observed in the I560T mutant is not due to the altered membrane expression but most likely due to the changes in channel gating properties.

\subsection{In silico simulation of KCNH2-I560T}

To explore whether the relatively modest gating modulation caused by the KCNH2-I560T mutation is sufficient to cause shortenings of APD and QT interval, we performed simulations of human ventricular action potentials with and without the KCNH2-I560T mutation in the 1-D myofiber model, representing the electrical behaviors of the left
A

$\mathrm{B}$
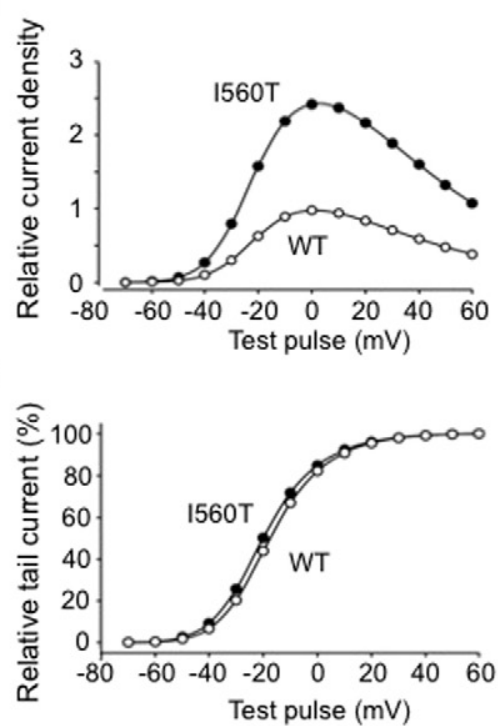

C

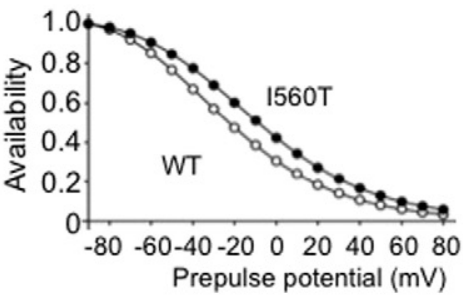

$\mathrm{D}$ Pacing $(1 \mathrm{~Hz})$

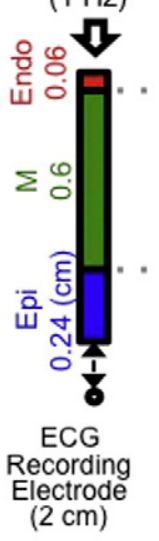

$(2 \mathrm{~cm})$
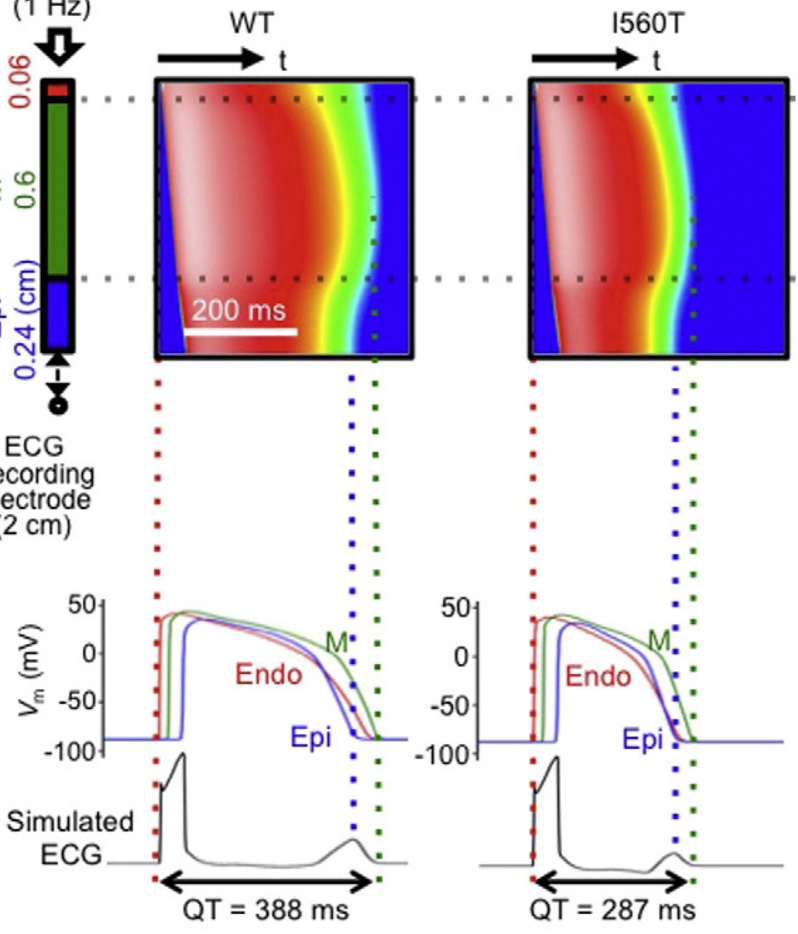

$\mathrm{E}$

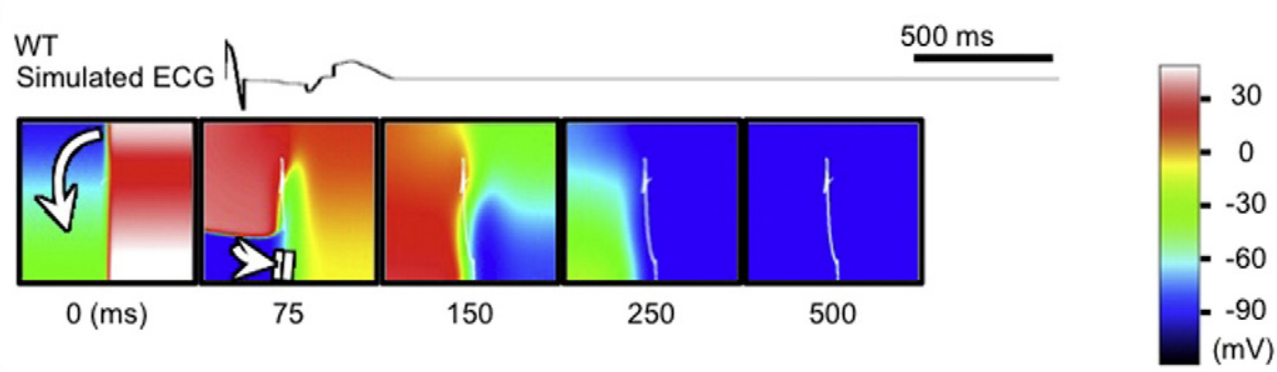

$\mathrm{F}$

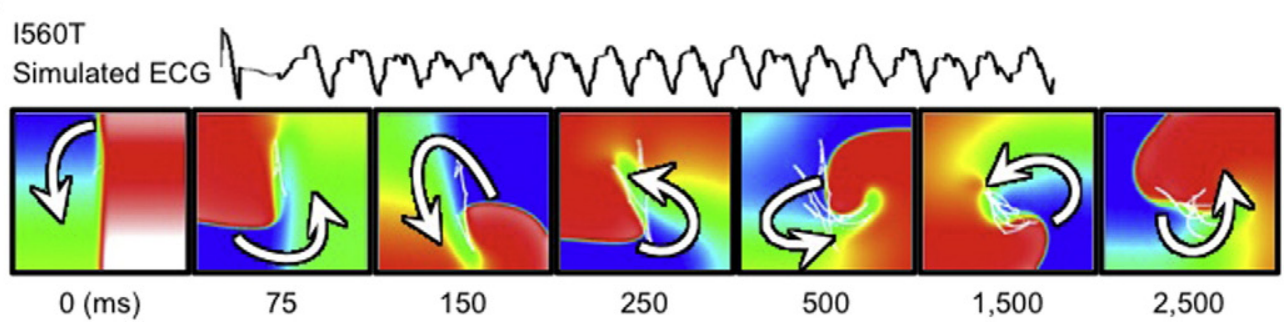

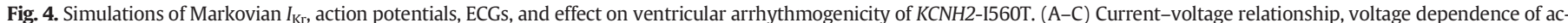

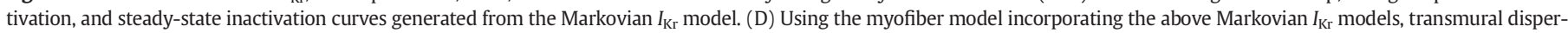

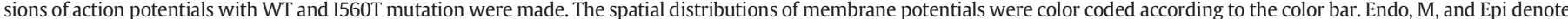

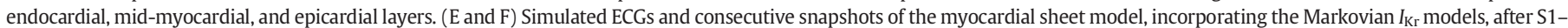

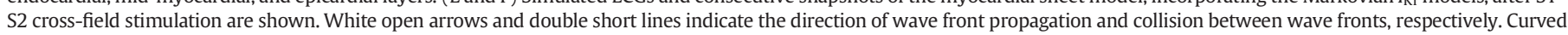
thin white lines inside the snapshots represent trajectories of the spiral wave phase singularities during the last $500 \mathrm{~ms}$ of activity. 
ventricular free wall. Using a modified computer model of Markovian $\mathrm{I}_{\mathrm{Kr}}$, we were able to faithfully reproduce the $\mathrm{I}_{\mathrm{Kr}}$ current traces and tail currents (Supplemental Fig. S2), 2.5-fold increase in current density (Fig. 4A), no significant shift in activation (Fig. 4B), and a $14 \mathrm{mV}$ positive shift in inactivation (Fig. 4C), consistent with our observations in vitro. When cells were stimulated at $1 \mathrm{~Hz}, K C N H 2-$ I560T showed significantly shorter APD than WT (Fig. 4D). The resulting pseudo-ECG also showed an abbreviated QT interval for the KCNH2-I560T mutant (287 ms) compared with WT (388 ms), which meets the diagnostic criteria of SQTS.

To explore the arrhythmogenic potency of VF in KCNH2-I560T, an S1-S2 cross-field protocol, with onset of S2 assumed as time zero ( $0 \mathrm{~ms}$ panel), was applied to induce a spiral wave re-entry (as a model of VF) (Fig. 4E,F). In the WT model, a counter-clockwise rotating wavefront terminated immediately (75-150 ms panels) and sustained re-entry was not induced. In contrast, the KCNH2-I560T model elicited a sustained meandering spiral wave re-entry with mean cycle length of 153 ms (Supplemental videos S1 and S2).

\subsection{Genotype-dependent differences in clinical characteristics of SQTS}

Based on the observations in our SQTS cohort, that KCNQ1 mutation carriers showed apparently earlier onset and more frequent bradyarrhythmia complications (Fig. 1, Table 1), we explored potential genotype-specific characteristics in the broader SQTS patient population. We combined mutation-positive SQTS patients of our cohort $(n=6)$ and those from previous publications $(n=59)$, and analyzed the clinical variables with respect to different genotypes (Tables 2 and 3). Among the SQT1, SQT2, and SQT3-6 groups, the age of manifestation was significantly later in SQT1 patients (SQT1: $35 \pm 19$ years, $\mathrm{n}=30$; SQT2: $17 \pm 25, \mathrm{n}=8$; SQT3-6: $19 \pm 15$, $\mathrm{n}=15$; $\mathrm{p}=0.011$ ), whereas the QTc values were comparable (Fig. 5, Table 2). Conversely, complications of SSS or bradycardia were significantly more prevalent in SQT2 patients $(6 / 8,75 \%)$ than non-SQT2 patients $(5 / 57,9 \%$; $<0.001)$ (Fig. 5C, Table 3), whereas there was no difference between SQT1 and non-SQT1 (Table 3). Furthermore, the prevalence of AF was also present in SQT2 patients $(5 / 8,63 \%)$ than non-SQT2 patients $(12 / 57,21 \%, \mathrm{p}=0.012)$ (Fig. 5D, Table 3 ). Other clinical parameters did not show significant differences between genotypes (Tables 2 and 3).

\subsection{Evaluation of penetrance in 16 SQTS families}

SQTS has been described as having close to complete penetrance in cohort studies, with only some exceptional cases with normal QTc [8, 9]. Despite carrying the mutation KCNQ1-V141M, the father of family 3 exhibited a QTc of $375 \mathrm{~ms}$, which is outside the diagnostic criteria for SQTS [20], but manifested chronic AF and bradycardia, prompting us to reevaluate the genetic penetrance of SQTS (Fig. 1C, Supplemental Fig. S1, D). Among 35 SQTS families, we focused on two families from our study and 14 previously reported families [1,3,5-9,12,14, 32-36] with two or more genetically or phenotypically affected individuals (Table 4). Our family 3 was the only SQTS family in this group carrying a KCNQ1 mutation. Among a total of 51 mutation-positive individuals, only 42 exhibited short QTc $<360$ ms. Therefore, the calculated overall genetic penetrance of SQTS was $82 \%$, which was lower than previously recognized $[8,9]$. Furthermore, we found that the 13 families with $\mathrm{K}$ channel mutations (SQT1-3) showed a higher penetrance of 90\%, whereas Ca channel mutations (SQT4-6) showed a much lower penetrance of 58\%. Interestingly, the low penetrance observed in families with Ca channel mutations is comparable to the well-known incomplete penetrance associated with Brugada syndrome [37]. Of the nine mutation carriers who did not exhibit short QTc, four K channel mutation carriers exhibited syncope, AF, bradycardia, or instances of nondocumented arrhythmia [35,36], and the five patients with Ca channel mutations remained asymptomatic [6,7].

\section{Discussion}

\subsection{Common electrophysiological properties in $\mathrm{KCNH} 2$ mutations}

The novel KCNH2 mutation I560T was identified in an SQT1 patient with severe QTc shortening, and family history of sudden death. Investigation of the KCNH2-I560T channel expressed in COS-7 cells showed a relatively mild gain of function with a $+14 \mathrm{mV}$ shift of steady-state inactivation but no activation abnormalities. The computer simulation recapitulated the APD shortening and susceptibility to ventricular reentry. Among four $\mathrm{KCNH} 2$ mutations that have been functionally evaluated, N588K was associated with a severe QTc shortening and exhibited severe gain of function with a 4 -fold increase in peak current density, virtually no inactivation over the physiological range, and a large positive shift $(+102 \mathrm{mV})$ of steady-state inactivation $[3,38]$. Peak current density was increased 6-fold in both T618I and E50D, and the steady-state inactivation was shifted by $+20 \mathrm{mV}$ and $+11.5 \mathrm{mV}$ in T618I and E50D, respectively $[8,14,39,40]$. Taken together, these results suggest that augmented peak current density and a positive shift of steady-state inactivation are the functional channel properties commonly affected by $K C N H 2$ mutations responsible for SQT1. The precise mechanism for the positive shift in inactivation curve in KCNH2-I560T is not clear, however, it is speculated that the shift in inactivation may be due to a disruption of the hydrogen bonding between amino acid residues that span the S5 and pore helix as seen in a neighboring residue H562 [41]. These changes in inactivation may be the primary determinants for the clinical manifestations of SQT1 [40]. However, as is observed in the SQT1 case carrying KCNH2-I560T reported here, the degree of the gating abnormality and the clinical severity may not always correlate, suggesting the involvement of additional confounding factors. These may include a number of common genetic variations that modulate QTc, as suggested by genome-wide association studies of LQTS $[42,43]$. Similar mechanisms may underlie the difference between clinical severity of SQTS patients and the electrophysiological properties of the mutant channels.

\subsection{Incomplete penetrance of SQTS}

Penetrance in LQTS has been recognized to be as low as $25 \%$ for some mutations [44], with the latent LQTS mutant carriers still, however, at risk of lethal arrhythmias and SCD [11]. By contrast, nearly complete penetrance has been described in SQTS within cohort studies [8,9]. However, our meta-analysis of 16 SQTS families revealed an incomplete penetrance of $82 \%$, where nine mutation-positive patients from six unrelated SQTS families exhibited longer QTc than $360 \mathrm{~ms}$ (Table 4). Responsible mutations for the latent SQTS cases include three K channel mutations: KCNQ1-V141M (family 3); KCNH2-E50D [8,36]; and KCNH2$\mathrm{R} 1135 \mathrm{H}$ [35]. The carriers of KCNQ1-V141M and $K C N H 2-\mathrm{R} 1135 \mathrm{H}$ showed arrhythmias in the absence of short QTc. Three other mutations

Table 2

Age of manifestation and QTc of SQTS patients.

\begin{tabular}{|c|c|c|c|c|c|}
\hline Clinical characteristics & SQT1 & SQT2 & SQT3-6 & $\mathrm{p}^{*}$ & Non-genotyped \\
\hline Age of manifestation (year) & $35 \pm 19(30)$ & $17 \pm 25(8)$ & $19 \pm 15(15)$ & 0.011 & $28 \pm 18(57)$ \\
\hline QTc (ms) & $307 \pm 30(31)$ & $305 \pm 33(8)$ & $329 \pm 55(21)$ & 0.107 & $311 \pm 27(65)$ \\
\hline
\end{tabular}

Mean \pm SD (n).

* Comparison between SQT1, SQT2, and SQT3-6. 
Table 3

Genotype-phenotype comparison SQTS patients.

\begin{tabular}{|c|c|c|c|c|c|c|c|}
\hline Clinical characteristics & SQT1 $(n=34)$ & non-SQT1 ${ }^{*}(\mathrm{n}=31)$ & $\mathrm{p} \dagger$ & SQT2 $(n=8)$ & non-SQT2 $\S(n=57)$ & $\mathrm{p} \ddagger$ & Non-genotyped $(\mathrm{n}=67)$ \\
\hline Male & $18(53 \%)$ & $12(39 \%)$ & 0.25 & $2(25 \%)$ & $28(49 \%)$ & 0.19 & 50 \\
\hline Syncope/palpitation & $8(24 \%)$ & $3(10 \%)$ & 0.123 & $0(0 \%)$ & $11(20 \%)$ & 0.17 & 8 \\
\hline SCD/aborted cardiac arrest & $6(18 \%)$ & $6(19 \%)$ & 0.86 & $2(25 \%)$ & $10(18 \%)$ & 0.6 & 20 \\
\hline $\mathrm{AF}$ & $8(24 \%)$ & $9(29 \%)$ & 0.614 & $5(63 \%)$ & $12(21 \%)$ & 0.012 & 11 \\
\hline SSS/bradycardia & $4(12 \%)$ & $7(23 \%)$ & 0.245 & $6(75 \%)$ & $5(9 \%)$ & $<0.001$ & 7 \\
\hline
\end{tabular}

$\mathrm{n}(\%)$. AF: atrial fibrillation, SCD: sudden cardiac death, SSS: sick sinus syndrome.

*Non-SQT1 denotes SQT2-6.

$\S$ Non-SQT2 denotes SQT1 and SQT3-6.

†Comparison between SQT1 vs SQT2-6.

$\ddagger$ Comparison between SQT2 vs SQT1, 3-6.

were found in Ca channel genes: CACNA1C-G490R, CACNB2-S481L and CACNA2D1-S755T. The carriers were asymptomatic [6,7]. The mechanisms underlying latent SQTS have not been determined; however, coexisting common polymorphisms that prolong repolarization may be potential candidates that mask the abbreviated QT intervals. In fact, among the mutant carriers of CACNA1C-G490R, an individual with a well-known KCNH2 polymorphism K897T [42] showed normal QTc, whereas the other two family members who only carry G490R manifested SQTS [6].

In SQT2, only three mutations (KCNQ1-V307L, -V141M, and -R259H) have to date been reported in de novo or sporadic cases $[4,9,13,15,16]$ and our family 3 carrying V141M is the first familial instance of SQT2. In view of the fact that the proband's father has exhibited chronic AF since 3 years of age without manifesting SQTS, despite carrying the V141M mutation, there may be additional latent carriers who do not show ECG abnormalities or other arrhythmias such as AF in the absence of QT shortening. Lack of familial SQT2 may be because the phenotypic manifestations of KCNQ1 mutations are milder than other subtypes, or because SQT2 has an extremely low penetrance. Further genetic screening of family members with non-remarkable ECG may help identify more latent $K C N Q 1$ carriers and better understand the natural history of SQTS.

\subsection{Genotype-specific clinical characteristics in SQTS}

The age of manifestation of mutation-positive SQTS patients spans from in utero to the eighth decade of life [8]. However, the agedistribution of lethal events in SQTS shows two peaks; one at the first year of life and another between 20 and 40 years of age [9]. Because we found that the age of initial clinical manifestation in SQT1 was significantly later than other subtypes, and six KCNQ1 mutation carriers exhibited apparent early onset of bradyarrhythmia, it is speculated that two distinct peaks of the first arrhythmic events may also be attributed to two genotypes (Fig. 5A, Table 2). A similar genotype-dependent age of manifestation is well known in LQTS; the majority of LQT2 (KCNH2) patients manifest their first symptoms after puberty, whereas LQT1

\section{A. Age of clinical manifestation}

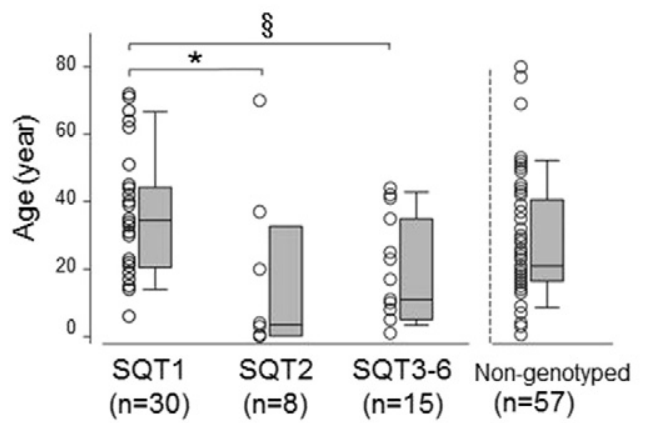

C. SSS and bradycardia

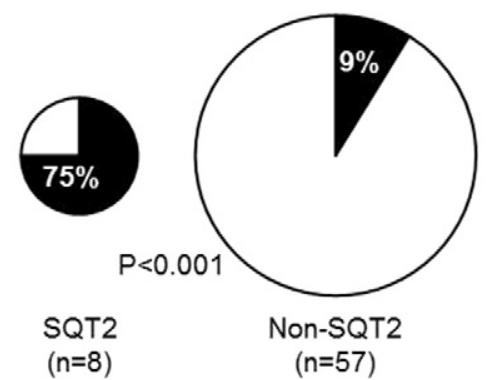

B. QTc

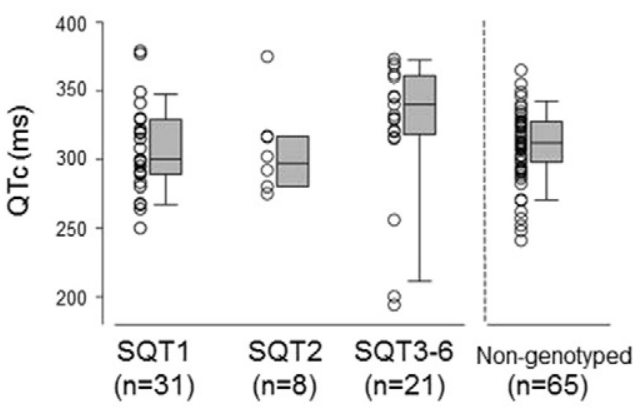

D. AF

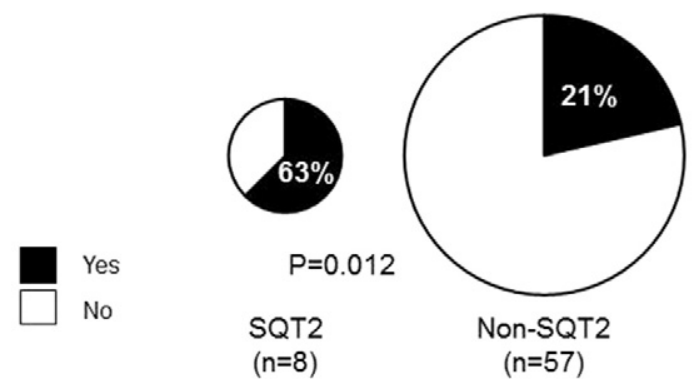

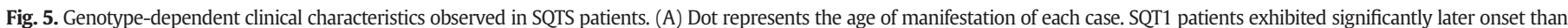

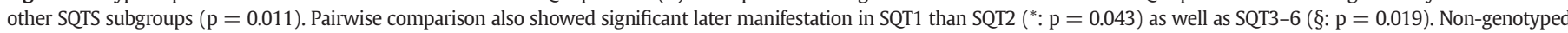

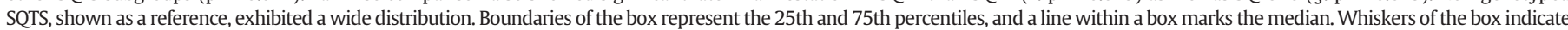

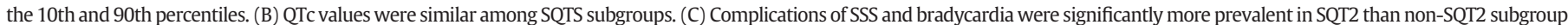
$(\mathrm{p}<0.001)$. (D) Complication of AF was significantly more prevalent in SQT2 patients than in non-SQT2 subgroup $(\mathrm{p}=0.012)$. 
Table 4

Mutations and the penetrance of 16 reported SQTS families.

\begin{tabular}{|c|c|c|c|c|c|c|c|}
\hline Family & Genes & Mutations & Affected (n) & Mutation-positive (n) & Penetrance (\%) & Other complications & References \\
\hline 1 & KCNH2 & N588K & 3 & 3 & 100 & - & {$[1,32]$} \\
\hline 2 & KCNH2 & N588K & 3 & 3 & 100 & - & {$[3,33,34]$} \\
\hline 3 & KCNH2 & N588K & 4 & 4 & 100 & - & {$[3,33,34]$} \\
\hline 4 & KCNH2 & N588K & 3 & 3 & 100 & - & [9] \\
\hline 5 & KCNH2 & $\mathrm{R} 1135 \mathrm{H}$ & 1 & 3 & 33 & (a), (b) & [35] \\
\hline 6 & KCNH2 & E50D & 1 & 2 & 50 & Syncope & {$[8,36]$} \\
\hline 7 & KCNH2 & T618I & 4 & 4 & 100 & - & [14] \\
\hline 8 & KCNH2 & T618I & 2 & 2 & 100 & - & [9] \\
\hline 9 & KCNH2 & T618I & 3 & 3 & 100 & - & Current study \\
\hline 10 & KCNH2 & Not reported & 6 & 6 & 100 & - & {$[12]$} \\
\hline 11 & KCNQ1 & V141M & 1 & 2 & 50 & $\mathrm{AF},(\mathrm{b})$ & Current study \\
\hline 12 & KCNJ2 & $\mathrm{D} 172 \mathrm{~N}$ & 2 & 2 & 100 & - & [9] \\
\hline 13 & KCNJ2 & D172N & 2 & 2 & 100 & - & [5] \\
\hline 14 & CACNA1C & G490R & 2 & 3 & 67 & - & [6] \\
\hline 15 & CACNB2 & S481L & 4 & 6 & 67 & - & [6] \\
\hline 16 & CACNA2D1 & S755T & 1 & 3 & 33 & - & [7] \\
\hline Total & & & 42 & 51 & $82 \%$ & & \\
\hline
\end{tabular}

(a): Non-documented arrhythmia, (b): bradycardia, AF: atrial fibrillation.

(KCNQ1) patients tend to become symptomatic before the age of 10 years [45].

We found a higher prevalence of bradyarrhythmia and AF in KCNQ1 mutant carriers than for other genotypes (Fig. 5C,D, Table 3). A gain of function in $\mathrm{I}_{\mathrm{KS}}$ has not only been associated with SQTS but also with familial AF [46] and sinus bradycardia [47]. This is thought to be primarily because the APD shortening occurs in the atrium as well as in the ventricle, increasing the susceptibility of atrial tissue to sustained re-entry [48]. Furthermore, computer simulations of the SQTS mutation, KCNQ1-V141M, and the familial AF mutation, KCNQ1-V241K, demonstrated that a gain of function in $\mathrm{I}_{\mathrm{Ks}}$ has been found to cause a cessation in spontaneous activity in the sinus node $[15,47]$. Such mechanisms may explain the observed phenotypic overlap and predominance of bradycardia and AF in SQT2.

\section{Study limitations}

In eight SQT2 patients we studied, six individuals carried the identical KCNQ1 mutation V141M. Therefore, the phenotype of the V141M mutation may be over-represented in our SQT2 data. As SQTS is a rare disease, the size of the population studied is small. Further delineation of this rare lethal arrhythmic syndrome warrants more extensive genetic and population studies using larger cohorts.

\section{Conclusions}

In summary, our study identified two $\mathrm{KCNH} 2$ mutations and one KCNQ1 mutation in five Japanese families with SQTS. The novel KCNH2-I560T mutation causes severe shortening of the QT interval and can trigger VF despite only a modest shift in inactivation. Among SQTS patients, there exist latent mutation carriers with ECG abnormalities such as AF and bradycardia indicating incomplete penetrance. Furthermore, despite the limited number of reported SQTS patients, our study suggests that clinical characteristics of SQTS can differ depending on the patient genotype, as is observed in LQTS.

Supplementary data to this article can be found online at http://dx. doi.org/10.1016/j.ijcard.2015.04.090.

\section{Conflict of Interest}

None.

\section{Acknowledgments}

We thank Dr. Tadashi Nakajima (Gunma University) for his assistance in sequencing our probands. We also thank Atsuko Iida, Saori Nakano, and Yasuko Noguchi for their technical assistance.

\section{Funding source}

This work was supported by Grant-in-Aid for Scientific Research on Innovative Areas (HD Physiology) 22136007, Grant-in-Aid for Scientific Research 24390199 from the Ministry of Education, Culture, Sports, Science and Technology, Japan, the Research Grant for the Cardiovascular Diseases (H24-033) from the Japanese Ministry of Health, Labour and Welfare (N.M.).

\section{References}

[1] I. Gussak, P. Brugada, J. Brugada, R.S. Wright, S.L. Kopecky, B.R. Chaitman, et al., Idiopathic short QT interval: a new clinical syndrome? Cardiology 94 (2000) 99-102.

[2] C. Patel, G.X. Yan, C. Antzelevitch, Short OT syndrome: from bench to bedside, Circ. Arrhythm. Electrophysiol. 3 (2010) 401-408.

[3] R. Brugada, K. Hong, R. Dumaine, J. Cordeiro, F. Gaita, M. Borggrefe, et al., Sudden death associated with short-QT syndrome linked to mutations in HERG, Circulation 109 (2004) 30-35.

[4] C. Bellocq, A.C. van Ginneken, C.R. Bezzina, M. Alders, D. Escande, M.M. Mannens, et al., Mutation in the KCNQ1 gene leading to the short QT-interval syndrome, Circulation 109 (2004) 2394-2397.

[5] S.G. Priori, S.V. Pandit, I. Rivolta, O. Berenfeld, E. Ronchetti, A. Dhamoon, et al., A novel form of short QT syndrome (SQT3) is caused by a mutation in the KCNJ2 gene, Circ. Res. 96 (2005) 800-807.

[6] C. Antzelevitch, G.D. Pollevick, J.M. Cordeiro, O. Casis, M.C. Sanguinetti, Y. Aizawa, et al., Loss-of-function mutations in the cardiac calcium channel underlie a new clinical entity characterized by ST-segment elevation, short QT intervals, and sudden cardiac death, Circulation 115 (2007) 442-449.

[7] C. Templin, J.R. Ghadri, J.S. Rougier, A. Baumer, V. Kaplan, M. Albesa, et al., Identification of a novel loss-of-function calcium channel gene mutation in short QT syndrome (SQTS6), Eur. Heart J. 32 (2011) 1077-1088.

[8] M.H. Gollob, C.J. Redpath, J.D. Roberts, The short QT syndrome: proposed diagnostic criteria, J. Am. Coll. Cardiol. 57 (2011) 802-812.

[9] A. Mazzanti, A. Kanthan, N. Monteforte, M. Memmi, R. Bloise, V. Novelli, et al., Novel insight into the natural history of short QT syndrome, J. Am. Coll. Cardiol. 63 (2014) 1300-1308.

[10] S.G. Priori, P.J. Schwartz, C. Napolitano, R. Bloise, E. Ronchetti, M. Grillo, et al., Risk stratification in the long-QT syndrome, N. Engl. J. Med. 348 (2003) 1866-1874.

[11] I. Goldenberg, S. Horr, A.J. Moss, C.M. Lopes, A. Barsheshet, S. McNitt, et al., Risk for life-threatening cardiac events in patients with genotype-confirmed long-QT syndrome and normal-range corrected QT intervals, J. Am. Coll. Cardiol. 57 (2011) $51-59$.

[12] C. Giustetto, R. Schimpf, A. Mazzanti, C. Scrocco, P. Maury, O. Anttonen, et al., Longterm follow-up of patients with short QT syndrome, J. Am. Coll. Cardiol. 58 (2011) 587-595. 
[13] J. Villafane, P. Fischbach, R. Gebauer, Short OT syndrome manifesting with neonatal atrial fibrillation and bradycardia, Cardiology 128 (2014) 236-240.

[14] Y. Sun, X.Q. Quan, S. Fromme, R.H. Cox, P. Zhang, L. Zhang, et al., A novel mutation in the KCNH2 gene associated with short QT syndrome, J. Mol. Cell. Cardiol. 50 (2011) 433-441.

[15] K. Hong, D.R. Piper, A. Diaz-Valdecantos, J. Brugada, A. Oliva, E. Burashnikov, et al., De novo KCNQ1 mutation responsible for atrial fibrillation and short QT syndrome in utero, Cardiovasc. Res. 68 (2005) 433-440.

[16] J. Villafane, J. Atallah, M.H. Gollob, P. Maury, C. Wolpert, R. Gebauer, et al., Long-term follow-up of a pediatric cohort with short QT syndrome, J. Am. Coll. Cardiol. 61 (2013) 1183-1191.

[17] M. Deo, Y. Ruan, S.V. Pandit, K. Shah, O. Berenfeld, A. Blaufox, et al., KCNJ2 mutation in short QT syndrome 3 results in atrial fibrillation and ventricular proarrhythmia, Proc. Natl. Acad. Sci. U. S. A. 110 (2013) 4291-4296.

[18] T. Hattori, T. Makiyama, M. Akao, E. Ehara, S. Ohno, M. Iguchi, et al., A novel gain-offunction KCNJ2 mutation associated with short-QT syndrome impairs inward rectification of Kir2.1 currents, Cardiovasc. Res. 93 (2012) 666-673.

[19] P. Maury, L. Hollington, A. Duparc, R. Brugada, Short QT syndrome: should we push the frontier forward? Heart Rhythm. 2 (2005) 1135-1137.

[20] S.G. Priori, A.A. Wilde, M. Horie, Y. Cho, E.R. Behr, C. Berul, et al., HRS/EHRA/APHRS expert consensus statement on the diagnosis and management of patients with inherited primary arrhythmia syndromes: document endorsed by HRS, EHRA, and APHRS in May 2013 and by ACCF, AHA, PACES, and AEPC in June 2013, Heart Rhythm. 10 (2013) 1932-1963.

[21] P. Syrris, A. Murray, N.D. Carter, W.M. McKenna, S. Jeffery, Mutation detection in long QT syndrome: a comprehensive set of primers and PCR conditions, J. Med. Genet. 38 (2001) 705-710.

[22] I. Splawski, J. Shen, K.W. Timothy, G.M. Vincent, M.H. Lehmann, M.T. Keating, Genomic structure of three long QT syndrome genes: KVLQT1, HERG, and KCNE1, Genomics 51 (1998) 86-97.

[23] N.M. Plaster, R. Tawil, M. Tristani-Firouzi, S. Canun, S. Bendahhou, A. Tsunoda, et al., Mutations in Kir2.1 cause the developmental and episodic electrical phenotypes of Andersen's syndrome, Cell 105 (2001) 511-519.

[24] P.S. Spector, M.E. Curran, A. Zou, M.T. Keating, M.C. Sanguinetti, Fast inactivation causes rectification of the IKr channel, J. Gen. Physiol. 107 (1996) 611-619.

[25] A. Kagan, Z. Yu, G.I. Fishman, T.V. McDonald, The dominant negative LOT2 mutation A561V reduces wild-type HERG expression, J. Biol. Chem. 275 (2000) 11241-11248.

[26] D. Shichi, T. Arimura, T. Ishikawa, A. Kimura, Heart-specific small subunit of myosin light chain phosphatase activates rho-associated kinase and regulates phosphorylation of myosin phosphatase target subunit 1, J. Biol. Chem. 285 (2010) 33680-33690.

[27] T. O'Hara, L. Virag, A. Varro, Y. Rudy, Simulation of the undiseased human cardiac ventricular action potential: model formulation and experimental validation, PLoS Comput. Biol. 7 (2011) e1002061.

[28] C.E. Clancy, Y. Rudy, Cellular consequences of HERG mutations in the long QT syndrome: precursors to sudden cardiac death, Cardiovasc. Res. 50 (2001) 301-313.

[29] I. Adeniran, M.J. McPate, H.J. Witchel, J.C. Hancox, H. Zhang, Increased vulnerability of human ventricle to re-entrant excitation in hERG-linked variant 1 short QT syndrome, PLoS Comput. Biol. 7 (2011) e1002313.

[30] T. Ashihara, T. Namba, T. Ikeda, M. Ito, M. Kinoshita, K. Nakazawa, Breakthrough waves during ventricular fibrillation depend on the degree of rotational anisotropy and the boundary conditions: a simulation study, J. Cardiovasc. Electrophysiol. 12 (2001) 312-322.

[31] M. Michaelsson, M.A. Engle, Congenital complete heart block: an international study of the natural history, Cardiovasc. Clin. 4 (1972) 85-101.

[32] K. Hong, P. Bjerregaard, I. Gussak, R. Brugada, Short QT syndrome and atrial fibrillation caused by mutation in KCNH2, J. Cardiovasc. Electrophysiol. 16 (2005) 394-396.

[33] C. Giustetto, F. Di Monte, C. Wolpert, M. Borggrefe, R. Schimpf, P. Sbragia, et al., Short QT syndrome: clinical findings and diagnostic-therapeutic implications, Eur. Heart J. 27 (2006) 2440-2447.

[34] F. Gaita, C. Giustetto, F. Bianchi, C. Wolpert, R. Schimpf, R. Riccardi, et al., Short QT Syndrome: a familial cause of sudden death, Circulation 108 (2003) 965-970.

[35] H. Itoh, T. Sakaguchi, T. Ashihara, W.G. Ding, I. Nagaoka, Y. Oka, et al., A novel KCNH2 mutation as a modifier for short QT interval, Int. J. Cardiol. 137 (2009) 83-85.

[36] C.J. Redpath, M.S. Green, D.H. Birnie, M.H. Gollob, Rapid genetic testing facilitating the diagnosis of short QT syndrome, Can. J. Cardiol. 25 (2009) e133-e135.

[37] C. Antzelevitch, P. Brugada, M. Borggrefe, J. Brugada, R. Brugada, D. Corrado, et al., Brugada syndrome: report of the second consensus conference, Heart Rhythm. 2 (2005) 429-440.

[38] J.M. Cordeiro, R. Brugada, Y.S. Wu, K. Hong, R. Dumaine, Modulation of I(Kr) inactivation by mutation N588K in KCNH2: a link to arrhythmogenesis in short QT syndrome, Cardiovasc. Res. 67 (2005) 498-509.

[39] A. El Harchi, D. Melgari, Y.H. Zhang, H. Zhang, J.C. Hancox, Action potential clamp and pharmacology of the variant 1 short QT syndrome T618I hERG $\mathrm{K}^{+}$channel, PLoS One 7 (2012) e52451.

[40] H.B. Martinez, D. Hu, M. Gollob, C. Antzelevitch, Novel gain-of-function N-terminal KCNH2 mutation associated with the short QT syndrome, Circulation 124 (2011) A12845.

[41] J.P. Lees-Miller, J.O. Subbotina, J. Guo, V. Yarov-Yarovoy, S.Y. Noskov, HJ. Duff, Interactions of H562 in the S5 helix with T618 and S621 in the pore helix are important determinants of hERG1 potassium channel structure and function, Biophys. J. 96 (2009) 3600-3610.

[42] L. Crotti, A.L. Lundquist, R. Insolia, M. Pedrazzini, C. Ferrandi, G.M. De Ferrari, et al, KCNH2-K897T is a genetic modifier of latent congenital long-QT syndrome, Circulation 112 (2005) 1251-1258.

[43] A. Pfeufer, S. Sanna, D.E. Arking M. Muller, V. Gateva, C. Fuchsberger, et al, Common variants at ten loci modulate the QT interval duration in the QTSCD Study, Nat. Genet. 41 (2009) 407-414.

[44] S.G. Priori, C. Napolitano, P.J. Schwartz, Low penetrance in the long-QT syndrome: clinical impact, Circulation 99 (1999) 529-533.

[45] P.J. Schwartz, S.G. Priori, C. Spazzolini, A.J. Moss, G.M. Vincent, C. Napolitano, et al., Genotype-phenotype correlation in the long-QT syndrome: gene-specific triggers for life-threatening arrhythmias, Circulation 103 (2001) 89-95.

[46] Y.H. Chen, S.J. Xu, S. Bendahhou, X.L. Wang, Y. Wang, W.Y. Xu, et al., KCNQ1 gain-offunction mutation in familial atrial fibrillation, Science 299 (2003) 251-254.

[47] C.S. Ki, C.L. Jung, H.J. Kim, K.H. Baek, S.J. Park, Y.K. On, et al., A KCNQ1 mutation causes age-dependant bradycardia and persistent atrial fibrillation, Pflugers Arch. 466 (2014) 529-540.

[48] J.C. Hancox, S. Kharche, A. El Harchi, J. Stott, P. Law, H. Zhang, In silico investigation of a KCNQ1 mutation associated with familial atrial fibrillation, J. Electrocardiol. 47 (2014) 158-165. 